\title{
The Extinction Behavior of Small Interacting Droplets in Cross-Flow
}

\author{
STEFANO RUSSO and ALESSANDRO GOMEZ* \\ Yale Center for Combustion Studies, Department of Mechanical Engineering, Yale University, \\ New Haven, CT, USA
}

\begin{abstract}
Experiments were conducted on monodisperse streams of small $(<100 \mu \mathrm{m})$ methanol and ethanol droplets, injected perpendicularly to the axis of an axisymmetric methane diffusion flame. After crossing the methane flame, the droplets ignited and continued to burn outside of the "domain of influence" of the gaseous flame till they eventually extinguished. The extinction behavior was studied as a function of droplet spacing, oxidizer concentration and velocity, and droplet velocity by measuring droplet size and velocity at the time of extinction using a commercial Phase Doppler Anemometer. The onset of droplet interaction effects on extinction was found at interdroplet distances well above the limits reported in the literature. The interaction is attributed to the vitiated atmosphere left behind by the leading droplet that affects the extinction conditions of the trailing one. The ratio between interdroplet time and convection time normal to the droplet direction of travel was found to be the controlling parameter. Results were correlated in terms of a critical Damköhler number that was found to scale as $d Y_{o, \infty}^{n_{o}} / v$, where $d$ is the droplet diameter, $Y_{o, \infty}$ is the oxidizer mass fraction, $v$ is the droplet velocity, and $n_{o}$ is 0.5 and 0.75 for methanol and ethanol, respectively. (C) 2002 by The Combustion Institute
\end{abstract}

\section{INTRODUCTION}

In many practical combustion systems such as oil burners, gas turbines, incinerators, diesel, and rocket engines, the liquid fuel is introduced in the combustion chamber as a spray of droplets. Different modes of burning can take place including external group combustion, in which a diffusion flame surrounds all vaporizing droplets inside the spray core, and internal group combustion, in which the primary flame is located inside the spray boundary, and some droplets may burn, individually or as a group, on the oxidizer side of the primary flame $[1,2]$. The transition from external group combustion to internal group combustion and, ultimately, to individual droplet burning can be promoted, for example, solely by increasing the oxygen concentration in the oxidizer stream [3]. The resulting change in the overall stoichiometry causes, in fact, an inward displacement of the primary diffusion flame and may result in the establishment of an internal group combustion regime, if the vaporization time is longer than the residence time available on the fuel side of the primary flame. In such a case, a droplet will pass through the reacting layer and burn isolated on

\footnotetext{
*Corresponding author. E-mail: alessandro.gomez@ yale.edu
}

the outside of the diffusion flame. In the case of turbulent environments, it is even possible that droplets with relatively short lifetimes might escape through the reacting layer following unusual time-temperature pathways [4].

Regardless of the mechanism by which isolated droplet burning occurs in a spray flame, these droplets are known not to burn to completion, but extinguish when they reach a critical diameter. As a result, emission of unburned hydrocarbons may ensue, which would be problematic, especially in the case of hazardous waste combustion, in which a fuel destruction efficiency in excess of $99.99 \%$ is required [5].

The extinction of a flame surrounding either a burning droplet or a porous sphere in a convective flow was studied both experimentally [6] and theoretically [7, 8]. Spalding [6, 7] was the first to show the linear behavior between velocity and size at extinction for large (3-6 $\mathrm{mm})$ porous spheres. More recently, the application of activation energy asymptotics [8] highlighted the existence of a critical Damköhler number for extinction. Computational work on droplet extinction also led to an interpretation of the results in terms of characteristic time scales and Damköhler number [9].

The objective of the present work is to examine interaction effects on droplet extinction in convective environments. To that end, monodis- 


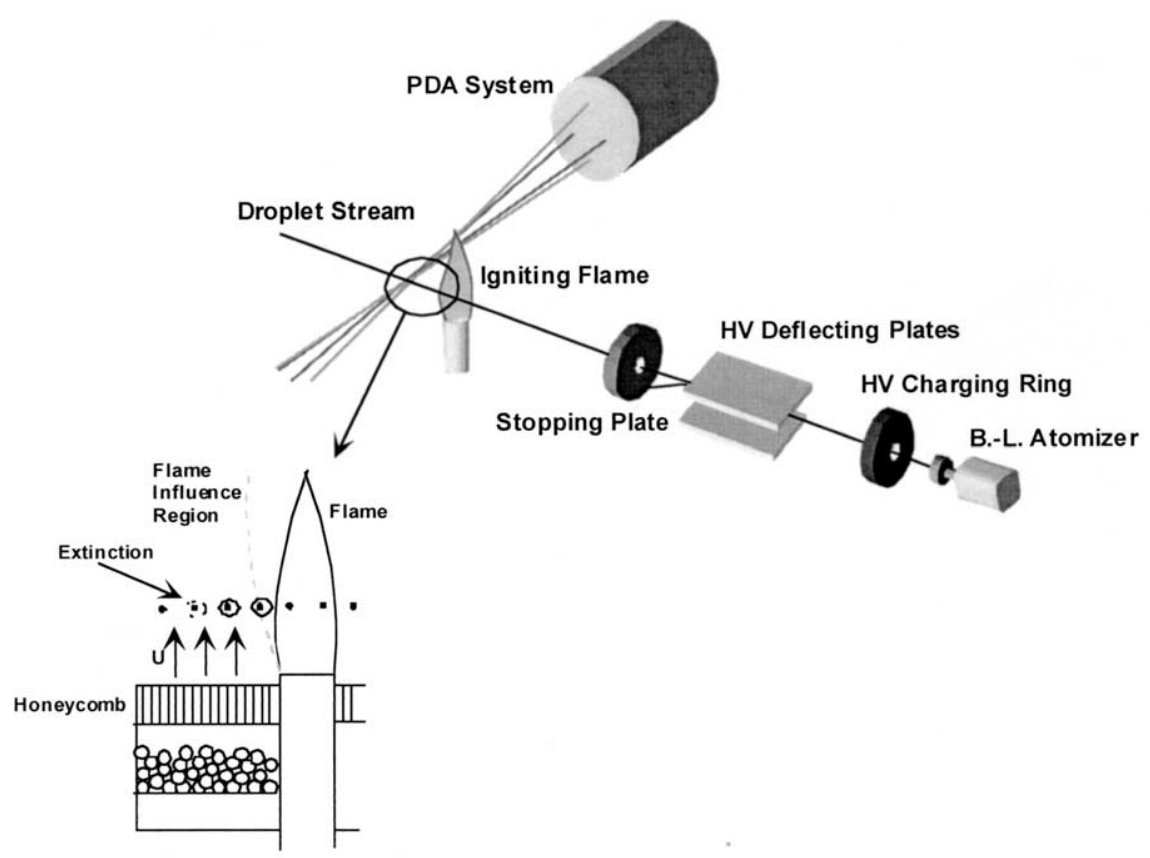

Fig. 1. Experimental apparatus.

perse streams of small ( $<100 \mu \mathrm{m}$ in diameter) alcohol droplets were injected perpendicularly to the axis of an axisymmetric methane diffusion flame. They ignited as they emerged from the flame, burned and subsequently extinguished. The array configuration provided a convenient and well-controlled way to carry out these interaction experiments and was widely used in the past to study flame structure [10] [11] [12], fluid dynamics interactions [13], ignition properties [14], and droplet burning time [15]. In the present study we identify the controlling parameter for the onset of interactive effects on the droplet extinction phenomenon and analyze the effect of the oxidizer mass fraction on the extinction of alcohol droplets.

\section{EXPERIMENTAL APPARATUS}

A sketch of the experimental apparatus is shown in Fig. 1. A co-flow laminar flame of methane and nitrogen is established at the mouth of a pipe measuring $11 \mathrm{~mm}$ in diameter by feeding $2.3 \mathrm{slpm}$ of nitrogen and $0.16 \mathrm{slpm}$ of methane in an oxidizer stream consisting of oxygen and nitrogen in various concentrations supplied on the outside of the pipe. A system of screens, packed glass beads, and honeycomb guarantees the laminarity of the oxidizer coflow. A monodisperse stream of either methanol or ethanol droplets of controlled size and spacing is injected normally to the methane flame. The droplets enter the flame, travel through it and are ignited at the exit by the reacting layer. The burning droplets continue to travel in the high temperature boundary layer of the gaseous flame for $\sim 4 \mathrm{~mm}$ before reaching the region unaffected by the flame. When flame extinction occurs in this region, size and velocity of the droplet at the instant of extinction are measured using a commercial Phase Doppler Anemometer (PDA) (Dantec Electronik). The extinction point is located by positioning the PDA probe volume at the point where chemiluminescence from the burning droplets ceases to be visible. An example of the measured droplet size and velocity distributions at extinction is shown in Fig. 2a and 2b. Because of trajectory effects that broaden artificially the size distribution, it is likely that the true size distribution is even narrower than in Fig. 2a [16]. The PDA, in the LDV mode, also provides the velocity of the oxidizer flow from $\mathrm{Al}_{2} \mathrm{O}_{3}$, seed particles of $1.5-\mu \mathrm{m}$ nominal diameter. Dis- 

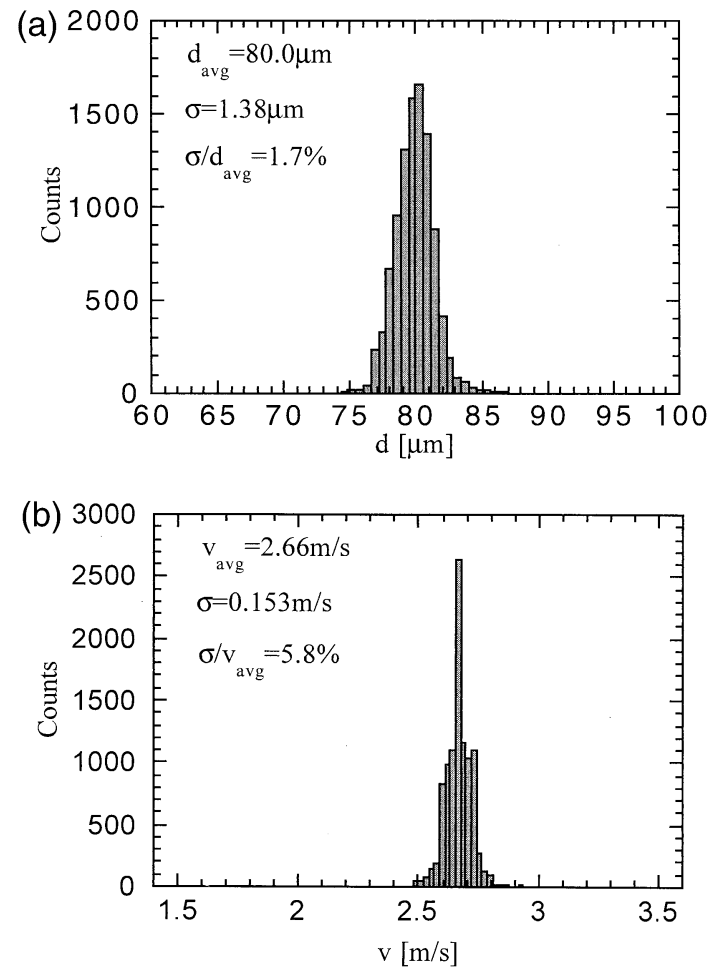

Fig. 2. a) Typical diameter histogram of extinguishing droplets; b)- Typical velocity histogram of extinguishing droplets.

crimination between droplet and particle velocities is obtained from the bimodality of the velocity distribution. Specifically, droplets present a large radial component and a very small axial component, whereas the opposite holds for the seed particles.

The method to generate the monodisperse droplet stream used in the present investigation is similar to the one implemented by Sangiovanni and Kesten [14]. A homemade vibrating orifice atomizer [17] provides a collimated, monodisperse stream of methanol droplets. A digitized function generator (Global Specialties 105-2003) provides the $25 \mathrm{~V}, 50 \mathrm{kHz}$ square wave needed to vibrate the $50-\mu \mathrm{m}$ orifice of the atomizer. The typical diameter of the generated droplets is calculated from the liquid flow rate and the applied frequency at $70 \mu \mathrm{m}$, with droplets as large as either $90 \mu \mathrm{m}$ or $114 \mu \mathrm{m}$ generated in a few cases. The rate at which droplets are injected into the flame is controlled by an electrostatic charging/deflection mechanism that periodically selects a droplet from the main stream. Differently from [14], the charging of the droplets is obtained by induction using a plate positioned at the jet break-up. The frequency at which droplets are extracted from the main stream varies from 50 to $500 \mathrm{~Hz}$. To achieve larger droplets and higher velocities, in some cases two consecutive droplets are selected from the main stream to enable them to coalesce and form a larger droplet with sufficient inertia to reach the measurement point at higher speed. The occurrence of the phenomenon has been verified using the PDA in "cold" measurements.

The relative position of the injection system and the flame is adjustable to achieve a broad range of extinction velocities for one setting of the droplet generation system. Atomizer, electrostatic system and burner are all mounted on a tri-dimensional translational stage to focus properly the PDA at the extinction point.

Table 1 lists the ranges of values of the following relevant parameters: the extinction diameter, the effective droplet generation frequency after the application of the deflection scheme, the extinction velocity, the oxidizer velocity and the oxygen mass fraction in the oxidizer stream. All data are for the experiments with methanol droplets. It should be noted that extinction diameters and velocities in columns 2 and 4 represent extinction "boundaries" originated from significantly different initial conditions.

Chemiluminescence and laser light scattering images of the extinguishing droplets are obtained using an intensified CCD camera (Santa Barbara Instruments ST-6B)

Lastly, the temperature field induced by the diffusion flame is measured using a coated

TABLE 1

Ranges of Values of Key Variables

\begin{tabular}{lccccc}
\hline Variable & $d_{\text {ext }}[\mu \mathrm{m}]$ & $\mathrm{f}[\mathrm{Hz}]$ & $v_{\text {ext }}[\mathrm{m} / \mathrm{s}]$ & $\mathrm{u}[\mathrm{m} / \mathrm{s}]$ & $\mathrm{Y}_{0, \infty}$ \\
\hline Range investigated & $40-90$ & $50-1000$ & $0.6-4.2$ & $0.15-0.50$ & $0.62-1.0$ \\
\hline
\end{tabular}




\section{$\longleftarrow$ Direction of droplet motion}

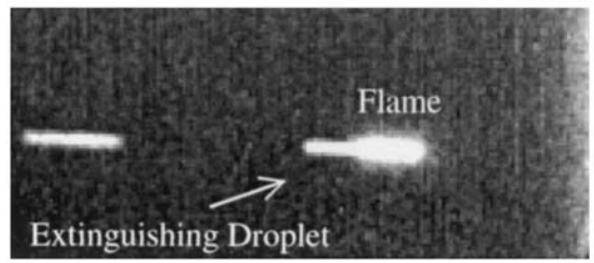

Fig. 3. Long exposure image of two consecutive droplets. Exposure time $400 \mu \mathrm{s}$. Oxidizer concentration at infinity $\mathrm{Y}_{\mathrm{o}, \infty}=0.71$. Droplet repetition rate $\mathrm{f}=900 \mathrm{~Hz}$.

$\mathrm{Pt} / \mathrm{Pt}-10 \% \mathrm{Rh}$ thermocouple with a bead of 200 $\mu \mathrm{m}$.

\section{RESULTS AND DISCUSSION}

As a droplet approaches the flame from the fuel side and passes through, if the conditions are favorable, it ignites, burns and eventually extinguishes. Figure 3 captures the last part of this sequence. It shows a long time exposure photograph of the streaks of two successive droplets traveling right to left with the droplets illuminated by a He-Ne laser sheet collinear with the droplet trajectory. The droplet in the middle of the picture is caught as it undergoes extinction, as can be inferred by the presence of a chemiluminescent "halo" in the right half of the streak. Clearly visible is the extinction point, when the luminosity from the flame ceases and the narrower cylindrical shape develops due solely to laser light scattering. The left half of that droplet image and the entire streak of the preceding droplet at the left of the image are made visible only by light scattering from the liquid phase, with no evidence of chemiluminescence.

In Fig. 4, we show normalized temperature and oxidizer concentration (left ordinate) versus time (lower abscissa) and space (upper abscissa), with the methane gaseous flame, assumed to be at the origin of both abscissae, burning in pure oxygen. The measurement location is $8 \mathrm{~mm}$ above the tip of the burner. The measured peak temperature is $1720 \mathrm{~K}$. No corrections for radiative losses were introduced, since the data are used primarily to show the qualitative behavior. The oxidizer concentration outside the reaction layer is estimated from the

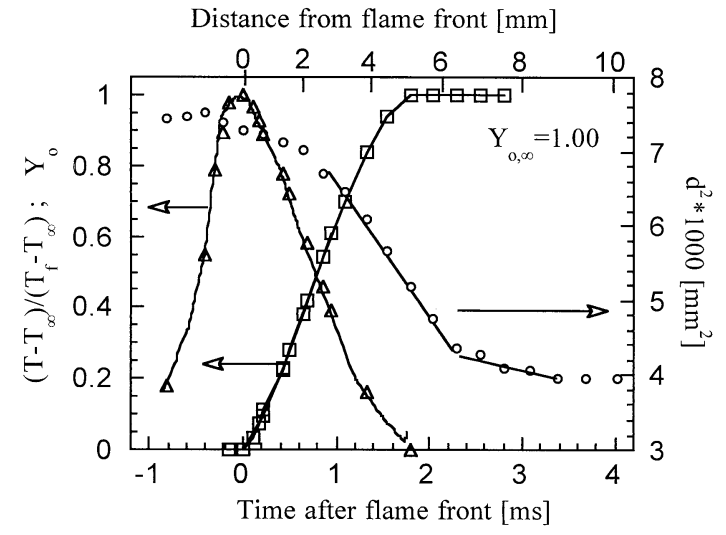

Fig. 4. Normalized temperature and oxidizer concentration (left ordinate) induced by the gaseous diffusion flame as a function of distance from the flame front (upper abscissa). $\mathrm{T}_{\mathrm{f}}=1720 \mathrm{~K}, \mathrm{~T}_{\infty}=290 \mathrm{~K}$. Square of the droplet diameter (right ordinate) as a function of the residence time after passing through the flame front (lower abscissa) or distance from the flame front (upper abscissa). Droplet diameter $=$ $86 \mu \mathrm{m}$; velocity $\mathrm{v}=3.2 \mathrm{~m} / \mathrm{s}$ in the reacting layer.

temperature profile, assuming Le $=1$ and the Burke-Schumann condition of $\mathrm{Y}_{\mathrm{O}}=0$ in the infinitely thin reaction layer.

Also plotted in Fig. 4 is the evolution of the square of the diameter of a sample droplet (right ordinate) as a function of time. The droplet, in this particular case $86 \mu \mathrm{m}$ in initial diameter and with a velocity of $3.2 \mathrm{~m} / \mathrm{s}$, after a small increase in size in the high temperature region of the gaseous flame, which can be attributed to the increasing liquid temperature, starts evaporating. After passing through the reacting layer, it burns surrounded by an envelope flame. It proceeds through a domain of influence of the methane gaseous flame, extending up to 4 to $5 \mathrm{~mm}$ away from the gaseous flame reacting layer, within which the igniting flame affects the conditions at "infinity" for the burning droplet, and, consequently its burning characteristics. The thickness of this domain of influence is regulated by a balance of convection along the $z$-axis and diffusion in the radial direction, as represented by the Peclet number, a non-dimensional parameter defined in terms of the oxidizer convective velocity, a reactant species diffusivity and a mixing layer thickness, $P e=u \delta / D$. In the ensuing discussion, even if the value of $u$ is changed, for all reported measurements extinction will always occur outside the methane flame domain of influence. 
Beyond the methane flame domain of influence, steady conditions of temperature and oxidizer concentration are encountered, as shown in Fig. 4. After a trajectory of $\sim 7 \mathrm{~mm}$ the diameter of the droplet, now down to $65 \mu \mathrm{m}$, stops decreasing marking the extinction of the envelope flame, at a velocity of $\sim 2.4 \mathrm{~m} / \mathrm{s}$.

We observe that after $1 \mathrm{~ms}$ the $\mathrm{d}^{2}$-law seems to be established for the burning droplet with vaporization constant that, in this particular case, is $1.75 \mathrm{~mm}^{2} / \mathrm{s}$. The extinction point is characterized by a change of slope indicating the abruptness of the extinction phenomenon, although a small amount of vaporization from residual heat in the droplet and gas-phase continues even after extinction, as previously remarked by Cho et al. [18]. The location where the diameter slope changes coincides with the end of visible flame chemiluminescence.

In the ensuing discussion we shall focus on those droplets which, after igniting in the reacting layer, travel in the flame influence region and extinguish outside such a region, that is, where temperature and oxidizer concentration at "infinity" have recovered the undisturbed value.

In a diffusive environment $\ell / d$, the interdroplet distance non-dimensionalized with respect to the droplet diameter, appears to be the critical parameter controlling droplet interaction. Even for arrays in convective conditions, this parameter has been used as a measure of droplet to droplet interaction $[14,15]$. Characteristic limiting values of $\ell$ for interaction to occur have been measured approximately to 20 to 30 droplet diameters [15]. However, droplet clouds are considered to be more effective in preventing single droplet burning, with values of interdroplet distance reaching 50 diameters in laminar sprays of monodisperse droplets [3].

We can postulate that in a convective environment the situation is sufficiently different from a diffusive situation, so that this geometric parameter should not be able to explain fully droplet interaction effects and departures from the single droplet behavior. For example, if we focus on the extinction point in the present experiments, an approaching burning droplet will find a vitiated environment left by the preceding extinguishing droplet, which will influence its extinction behavior unless enough time is given to the oxidizer cross-flow, at velocity $\mathrm{u}$, to sweep out the degraded layer and regenerate the oxidizing atmosphere at the oxidizer feed stream composition and temperature. Of course, whether this regenerative effect is due mostly to convection or a combination of diffusion and convection depends on the specific experimental conditions.

A simple criterion to understand when there is interaction between droplets can be established by comparing two characteristic times. The first, the interdroplet time, $t_{\text {int }}$, is the transit time between two successive droplets, and can be defined as the inverse of the droplet repetition rate $t_{\mathrm{int}}=\frac{1}{f}$. The second is the washout time, $t_{w o}$, that is, the time needed for the oxidizer flow to wash out the vitiated atmosphere left behind by the leading droplet, under the tacit assumption to be corroborated by the experiments that convection dominates. The latter can be defined as the ratio between the flame size, estimated at 5 times the droplet diameter for the present methanol/oxygen stoichiometry, and the oxidizer velocity: $t_{w o} \approx \frac{5 d}{u}$. It is a measure of the convective time in the direction normal to the droplet trajectory.

The ratio of these two times is $\Lambda=\frac{t_{\text {int }}}{t_{w o}}$ $\approx \frac{u}{5 d f}$. We can postulate that there will be a critical value $\Lambda_{\mathrm{c}}$, such that if $\Lambda<\Lambda_{\mathrm{c}}$ the leading droplet will influence the trailing one and droplet interaction effects should set in. Conversely, if $\Lambda>\Lambda_{\mathrm{c}}$ single droplet behavior should be recovered, that is, each droplet burns surrounded by its own flame and without any influence on the surrounding environment from the preceding droplet in the stream. It is worthwhile remarking that $\Lambda$ can be rewritten as $\Lambda=\frac{1}{5} \frac{\ell}{d} \frac{u}{v}$, where $v$ is the droplet velocity. Thus, the controlling parameter is the non-dimensional interdroplet distance multiplied by a velocity correction factor accounting for convective effects. In light of these premises, the experimental results will be presented as these three parameters, droplet size, oxidizer velocity and droplet frequency, are varied within the 


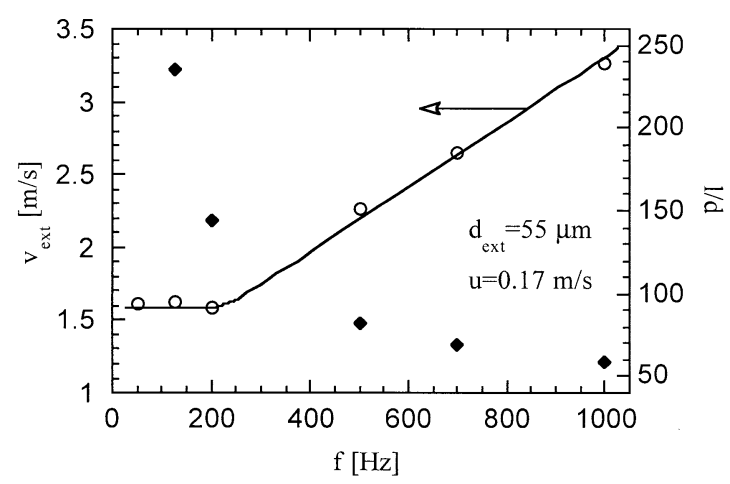

Fig. 5. Extinction velocity (left ordinate) and non-dimensional inter-droplet distance (right ordinate) for droplets with extinction diameter of $55 \mu \mathrm{m}$ droplets as a function of the droplet repetition rate at constant oxidizer cross-flow velocity.

ranges specified in Table 1 for fixed oxidizer concentration.

Figure 5 shows the change in extinction velocity (left ordinate) as a function of the droplet frequency for droplets extinguishing with a diameter of $55 \mu \mathrm{m}$ in a $0.17 \mathrm{~m} / \mathrm{s}$ cross-flow of pure oxygen. We note that by extinction velocity and extinction diameter we refer to the values of these variables when the flame surrounding the droplet goes out. We change conditions upstream at will, ensuring that the droplets would cross the methane flame, ignite, and extinguish outside the gaseous flame domain of influence. If these conditions are fulfilled, all the data are correlated independently of the initial conditions. The extinction velocity is initially constant at around $1.6 \mathrm{~m} / \mathrm{s}$ and independent of frequency up to approximately $\mathrm{f}=200 \mathrm{~Hz}$. For higher frequencies, it varies linearly with frequency. This latter behavior can be explained in terms of the dimensionless parameter governing the extinction of a single droplet, the Damköhler number, representing the ratio between a characteristic flow time and a chemical time. For a droplet in a convective environment, the flow time is on the order of $\mathrm{t}_{\mathrm{m}}=d / v$, where $d$ and $v$ represents diameter and velocity, respectively. Such a time plays the role of the inverse of the strain rate in the canonical counter-flow diffusion flame theory. At extinction, this time is on the same order of the chemical time. The increase of extinction velocity with frequency in Fig. 5 suggests the capability of the droplet to withstand higher strain rates before extinguishing. In effect, it can be interpreted as a reduction of the extinction Damköhler number since the preceding droplet in the trail "assists" the burning of the subsequent one. For example, the leading and extinguishing droplet may contribute to the combustion of the trailing one some residual heat in the gas-phase and some fuel vapor. In a regime of vigorous burning bringing droplets close to one another may hinder combustion, which has a simple masstransport interpretation, as the droplets compete for the deficient reactant, the oxidizer, leading to the onset of group combustion. In contrast, here we examine conditions of "group extinction" in which the deficient reactant is the fuel and competition for oxygen is no longer controlling. Rather, starvation of fuel in the gaseous phase controls, and extinction is delayed by any process that helps increasing the presence of fuel in the vapor phase, such as the puff of fuel vapor from the preceding droplet in the array after it has extinguished.

These results are consistent with the anticipated role of $\Lambda$, suggesting that for $\mathrm{f}<200 \mathrm{~Hz}$, or, equivalently, $\Lambda>\Lambda_{\mathrm{c}}$, single droplet burning is observed, whereas in the region of larger frequencies $\left(\Lambda<\Lambda_{\mathrm{c}}\right)$ droplet interaction affects the extinction behavior. In the same figure we also plot (right ordinate) the interdroplet distance, non-dimensionalized with respect to the droplet diameter, $l / d$. The spacing between the droplets is much larger than the one usually considered maximal for interactions to take place $(\sim 30 d)$, which indeed confirms the significant role of forced convection in the present situation. In fact, the velocity correction factor, $u / v$, in the controlling parameter $\Lambda$ takes values ranging between 0.1 to 0.2

The presence of a critical value of $\Lambda$ can be inferred by examining Fig. 6, showing the change in extinction velocity as function of the parameter $\Lambda$ for three different values of the droplet extinction diameter. For each droplet size, $\Lambda$ was varied by increasing the oxygen co-flow normal to the droplet trajectory. In all three cases, it appears that a critical value of $\Lambda_{\mathrm{c}}$ $\approx 3.1$ is obtained beyond which there is no change in the extinction velocity and we can assume that the trailing droplet is not influenced anymore by the leading one. 


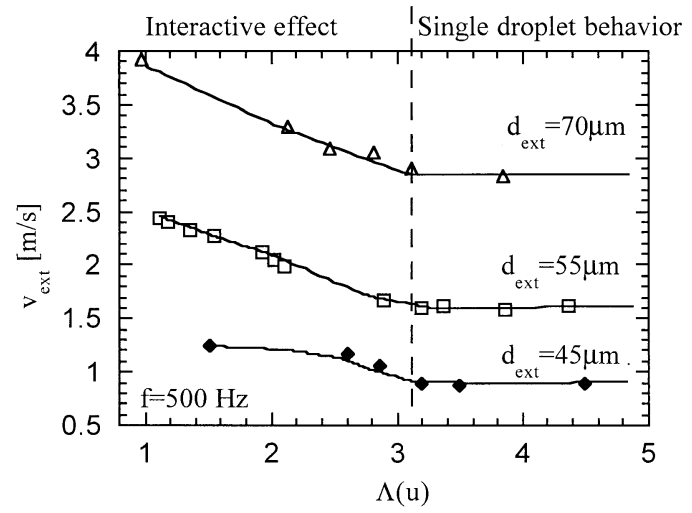

Fig. 6. Extinction velocity as a function of the $\Lambda$ parameter for three different extinction diameters and constant droplet repetition rate.

Let us now focus our attention on the diameter component of the parameter $\Lambda$. In Fig. 7 we show the behavior of extinction velocity vs. droplet extinction diameter for different frequencies and oxidizer velocities. For fixed conditions of frequency and oxidizer velocity there is a critical diameter below which no interaction is expected $\left(\Lambda>\Lambda_{\mathrm{c}}\right)$. For example, when $\mathrm{f}=$ $100 \mathrm{~Hz}$ and $\mathrm{u}=0.17 \mathrm{~m} / \mathrm{s}$, if we assume $\Lambda_{\mathrm{c}} \approx 3.1$, the critical value of the diameter is $d_{c}$ $=\frac{u}{5 \Lambda_{c} f} \approx 110 \mu m$, well outside the area currently under investigation. As a result, the open diamonds represent the behavior of noninteracting droplets. Therefore, we can assume the straight line for $\mathrm{f}=100 \mathrm{~Hz}$ and $\mathrm{u}=0.17 \mathrm{~m} / \mathrm{s}$ to be representative of the extinction velocity vs. extinction diameter for non-interacting droplets

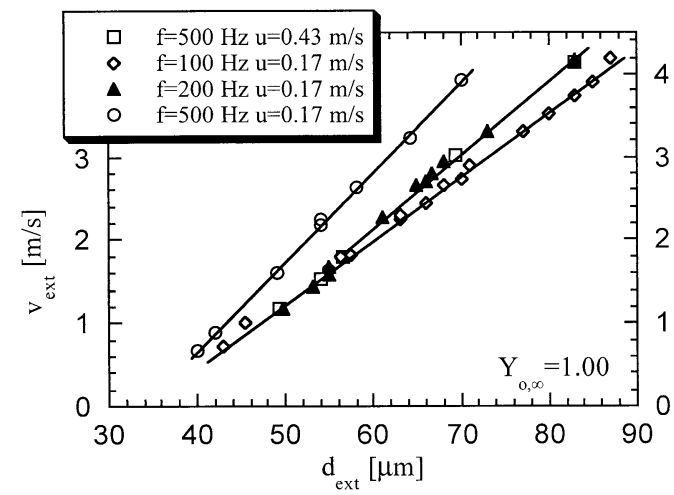

Fig. 7. Extinction velocity as a function of droplet diameter for different conditions of oxidizer velocity $\mathrm{u}$, and droplet repetition rate $\mathrm{f}$. in the range 30 to $90 \mu \mathrm{m}$. Vice versa, if we examine the case $\mathrm{f}=500 \mathrm{~Hz}$ and $\mathrm{u}=0.17 \mathrm{~m} / \mathrm{s}$, we obtain $d_{c}=\frac{u}{5 \Lambda_{c} f} \approx 22 \mu \mathrm{m}$. This value is well below the range in Fig. 7. So we can assume that for these conditions the leading droplet always influences the trailing one. As a result, the open circles in Fig. 7 pertain to extinction behavior vitiated by droplet interaction.

The anticipated behavior is even confirmed at intermediate conditions, when $\mathrm{f}=200 \mathrm{~Hz}$ and $\mathrm{u}=0.17 \mathrm{~m} / \mathrm{s}$ (full triangles), which yield $d_{c}=\frac{u}{5 \Lambda_{c} f} \approx 55 \mu m$. The extinction velocity in this case seems to follow the behavior of the non-interacting line until approximately $d=55$ $\mu \mathrm{m}$, that is, the data overlap the line of the open diamonds. At this point, however, droplet interaction effects start, the curve diverges and extinction is observed at higher velocities as compared to the non-interacting line. A similar behavior is obtained if we increase frequency and oxidizer velocity by the same factor, that is, if we keep $d_{c}$ constant. This is the case shown with open squares, corresponding to $\mathrm{f}=500 \mathrm{~Hz}$ and $\mathrm{u}=0.43 \mathrm{~m} / \mathrm{s}$. As expected also in this case the critical diameter appears to be around $d=$ $55 \mu \mathrm{m}$.

It should be remarked that the "cold" Reynolds number for this range of size and velocities is comprised approximately between 2 and 40, which is not sufficiently large for droplets to experience a transition from envelope to wake flame [9, 19], which would have compromised the linear relation between diameter and velocity. Chemiluminescence images taken with an intensified CCD camera, and not reported here, confirmed this hypothesis.

The correlation in Fig. 7 clearly points to a dominant role of convection over diffusion, as we initially postulated. This result can be rationalized as follows.

First, an upper estimate of the diffusion velocity is given by

$$
-D \cdot \frac{\nabla Y_{F}}{Y_{F}} \approx \frac{D \cdot}{5 d} \frac{\Delta Y_{F}}{Y_{F}} \approx \frac{1.5 \cdot 10^{-5}}{5 \cdot 60.10^{-6}} \approx .05 \mathrm{~m} / \mathrm{s}
$$

that is, the diffusivity over the size of the fuel region left behind by the extinguished droplet, of the order of the flame volume, where we have 
assumed $\Delta Y_{F} / Y_{F}$ to be of order unity initially. This value should be compared with a typical convective velocity of the cross flow of $0.17 \mathrm{~m} / \mathrm{s}$ in the experiments. $\Delta Y_{F} / Y_{F}$ remains of order unity when there is a droplet in the core of the puff that continuously provide a source of fuel vapor. Not so after extinction, when the droplet and the existing fuel puff will separate because of their difference in mass (inertia). Moreover, the droplet leaves fuel vapor behind only at the beginning. It stops vaporizing after a brief thermal transient during which it cools down. So, the extinct droplet keeps barreling along while the fuel vapor puff is left behind to face the sweeping cross-flow $\mathrm{u}$. Because the total mass of the fuel puff must stay constant, once it is separated from the source, the diffusion velocity decays in time for the diminishing concentration gradients resulting from the coupled effects of diminished $\Delta Y_{F} / Y_{F}$ and increasing length scale. On the other hand, convection sweeps away leftovers cleanly and abruptly. So, although diffusion may not be always negligible, convection appears to dominate, as our correlation suggests.

Second, if the interaction between two droplets can be interpreted thermally, as an energy input into the trailing droplets from the combustion products left behind from the preceding and extinct droplet, the characteristic length scale is a multiple of the droplet diameter greater than the estimated stand-off ratio, since hot combustion products are present also on the outside of the individual flame.

Third, the length scale $5 \mathrm{~d}$ is based on the theoretical flame stand-off ratio from the classical purely diffusive theory. From pictures of the chemiluminescent region, the size of the visible flame appeared close to $0.5 \mathrm{~mm}$, that is, closer to a 10d. Replacing such a lengthscale in the expression of the diffusion velocity above would halve its value and make the convective argument even more compelling.

All the results presented so far pertained to a fixed unity oxidizer mass fraction. We can now examine the effect of the oxidizer mass fraction on the extinction behavior. The results are correlated in Fig. 8, showing the extinction velocity as a function of the product of the droplet diameter at extinction times the square root of the oxygen mass fraction. The data

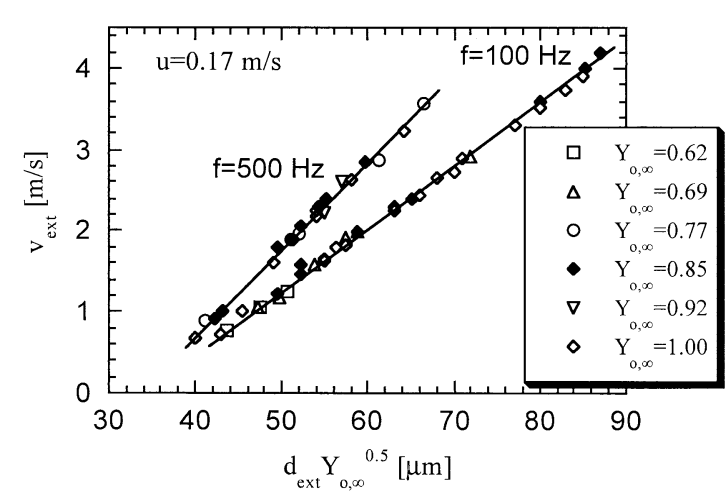

Fig. 8. Extinction velocity for methanol droplets at two different repetition rates as function of the product of droplet diameter times the square root of the oxidizer mass fraction. The oxidizer velocity is held constant at $\mathrm{u}=0.17$ $\mathrm{m} / \mathrm{s}$.

collapse around two lines, one obtained for $\mathrm{f}=$ $100 \mathrm{~Hz}$ and $\mathrm{u}=0.17 \mathrm{~m} / \mathrm{s}$, which, as discussed in connection with Fig. 7, relates to single droplet behavior, the other, obtained for $\mathrm{f}=500 \mathrm{~Hz}$ and $\mathrm{u}=0.17 \mathrm{~m} / \mathrm{s}$, relating to droplet interactive behavior (see also Fig. 6). The data cover droplet sizes in the range 40 to $90 \mu \mathrm{m}$ with velocities between $0.7 \mathrm{~m} / \mathrm{s}$ and $4.5 \mathrm{~m} / \mathrm{s}$ and oxygen mass fraction spanning the range 0.62 through 1.00. Limitations in the droplet generation capabilities of the atomizer and in the radial dimension of the oxidizer stream prevented us from exploring an even broader range.

The success of this correlation prompts us to interpret the results in terms of a critical Damköhler number at extinction. If the chemical time is defined as

$t_{c h}^{-1}=\beta\left[\mathrm{CH}_{3} \mathrm{OH}\right]^{n_{F}}\left[\mathrm{O}_{2}\right]^{n_{o}} \exp \left(-\frac{E_{a}}{R T}\right)$

and fuel and oxidizer concentrations are reinterpreted in terms of mass fractions, the Damköhler number at extinction, $\mathrm{Da}_{\mathrm{c}}$, is:

$D a_{c}=\frac{t_{m}}{t_{c h}} \propto \frac{d}{\tilde{v}} Y_{F, d}^{n_{f}} Y_{O, \infty}^{n_{o}} \frac{1}{T^{n_{F}+n_{o}}} \exp \left(-\frac{E_{a}}{R T}\right)$

Where $\tilde{v}$ is a rescaled velocity $\tilde{v}=v-v_{0}$, and $v_{0}$ $=-2.5 \mathrm{~m} / \mathrm{s}$ is the extrapolated value of the velocity at $d=0$.

The correlation in Fig. 8 is consistent with the concept of a constant extinction Damköhler 


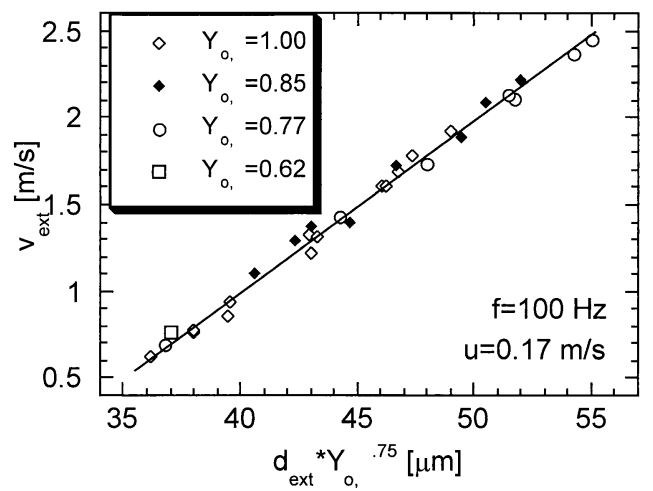

Fig. 9. Extinction velocity for ethanol droplets at two different repetition rates as function of the product of droplet diameter times the oxidizer mass fraction to the 0.75 power. Oxidizer velocity and repetition rate are held constant at $\mathrm{u}=0.17 \mathrm{~m} / \mathrm{s}$ and $100 \mathrm{~Hz}$, respectively.

number, provided that $\mathrm{n}_{\mathrm{O}}=0.5$ and the temperature dependent term is approximately constant. Note that the exponent of the oxidizer mass fraction remains at 0.5 even in the case in which droplet interaction effects are present (upper curve). This value, perhaps fortuitously, is in agreement with the oxidizer exponent found in shock tube experiments [20].

Similar studies have been carried also for the extinction of small Ethyl Alcohol droplets. In Fig. 9 we report extinction velocity as function of $d \cdot Y_{o}^{0.75}$. The correlation for different oxidizer concentrations is excellent, confirming that the overall kinetics of ethyl alcohol is approximately of order 0.75 with respect to the oxidizer concentration. Even in this case this value agrees with the oxidizer exponent found in shock tube experiments [21].

If the correlations in Figs. 8 and 9, are to be interpreted in terms of a critical Damköhler, the implication of the constancy of the temperature-dependent term is rather surprising, since the change in oxidizer concentration of those experiments should result in higher flame temperatures. A plausible explanation for the constancy of the temperature-dependent term may be offered by the following consideration. For liquid fuels the flame temperature is affected by the presence of the droplet that acts as a constant temperature sink for the heat generated in the flame. The higher the oxygen mass fraction, the closer the flame gets to the droplet increasing the heat flux into the droplet. This droplet sink effect can further contribute to offset the anticipated increase in flame temperature subsequent to an increase in oxidizer concentration. This effect may be particularly significant for alcohols because of the high value of the ratio of the enthalpy of vaporization to the enthalpy of combustion $\left(50 \cdot 10^{-3}\right.$ compared with, say, $6.7 \cdot 10^{-3}$ for heptane), which results in steeper temperature gradients on the droplet side of the flame and larger heat fluxes as compared to hydrocarbons [22]. Unfortunately, we could not test this point experimentally The charging device can work either with polar liquids (e.g., alcohols) or with liquids with finite electric conductivity, unlike hydrocarbons. Doping hydrocarbons with a suitable additive, which we tried, does not work, since it requires concentration levels that ultimately lead to microexplosions, thereby affecting the sensitive extinction condition.

A potentially important role of water vapor absorption in the extinction of alcohol droplets has been proposed in [18, 23, 24]. We could not verify it experimentally. However, it is likely that, because burning occurred in a very short time in view of the small size of the droplets and the large oxygen concentration, water vapor absorption plays a minor role. In fact, the effect appears to be significant mostly for large droplets and long vaporization time, since the absorbed water vapor at the surface of the droplets is required to diffuse into the interior [23]. Because Peclet numbers for the liquid phase have values on the order of 100, this effect is not significant. Nor could recirculation in the droplet interior help. In fact, the ratio of a diffusion time in the liquid droplet to a hypothetical turnover time of a vortex within a droplet, if such a circulation existed, can be shown to be equal to the droplet Reynolds number times the ratio of the kinematic viscosity of the gas to the kinematic viscosity of the liquid. At the prevailing Reynolds number, in the range 2 to 40 , the ratio of these two times is of order one and recirculation could not change these estimates dramatically. Nor could a possible recirculation because of the droplet generation scheme linger in the combustion region, since it would be attenuated by viscous effects over characteristic times shorter than the transient time across part of the cross flow oxygen 
stream and the gaseous methane flame before droplet ignition. We conclude that water vapor absorption should not be relevant in the present experimental conditions.

\section{CONCLUSIONS}

This study demonstrates that in arrays of monodisperse droplets, interaction effects on extinction in convective flows, appear at interdroplet distances well above the limits reported in literature. The effect is attributed to the vitiated atmosphere left behind by the leading droplet that affects the extinction conditions of the trailing one. Droplet interaction effects set in when the ratio between interdroplet time and convection time, $\Lambda$, attains a critical value. $\Lambda$ is equivalent to the widely used non-dimensional interdroplet distance multiplied by a factor representing the ratio between droplet velocity and oxidizer cross-flow velocity. When $\Lambda$ reaches a critical value, measured to be $\sim 3.1$, droplet interaction effects cease to exist and single droplet behavior is recovered. The measured extinction velocities have been correlated in terms of a critical extinction Damköhler number that was found to scale as $d Y_{o, \infty}^{n_{o}} / v$, with exponent equal to 0.5 and 0.75 for methanol and ethanol droplets, respectively.

The support of NSF, Grant \# CTS-9904296 (Dr. Farley Fisher, Contract Monitor), is gratefully acknowledged. The authors wish to thank Professor Amable Liñàn for helpful discussions.

\section{REFERENCES}

1. Chiu, H. H., and Liu, T. M., Combust. Sci. Tech. 17:127 (1977).

2: Labowsky, M., and Rosner, D. E., in Advances in Chemistry Series 166: Evaporation-Combustion of Fuels (J. T. Zung, Ed.) American Chemical Society, Washington, D.C., 1978. p. 63.

3. Chen, G., and Gomez, A., Combust. Flame 110:392 (1997).

4. Kramlich, J. C., Randall Seeker, W. M., and Sam- uelsen G. S., Twenty-First Symposium (International) on Combustion, The Combustion Institute, Pittsburgh, 1986, p. 593.

5. Mulholland, J. A., Srivastava, R. K., Wendt, J. O. L., Agrawal, S. R., and Lanier, W. S., Combust. Flame 86:297 (1991).

6: Spalding, D. B., Fourth Symposium (International) on Combustion, The Combustion Institute, Pittsburgh, 1954, p. 255.

7. Spalding, D. B., Fuel 33:255 (1954).

8. Wu, X., Law, C. K., and Fernandez-Pello C., Combust. Flame 44:113 (1982).

9. Dwyer, H. A., and Sanders, B. R., Twenty-First Symposium (International) on Combustion, The Combustion Institute, Pittsburgh, 1986, p. 633.

10. Zhu, J. Y., and Dunn-Rankin, D., Twenty-Fourth Symposium (International) on Combustion, The Combustion Institute, Pittsburgh, 1992, p. 1473.

11. Sangiovanni, J. J, and Dodge, L. G., Seventeenth Symposium (International) on Combustion, The Combustion Institute, Pittsburgh, 1978, p. 455.

12. Miyasaka, K., and Law, Chung K., Eighteenth Symposium (International) on Combustion, The Combustion Institute, Pittsburgh, 1981, p. 283.

13. Tal, R., Lee, D. M., and Sirignano, W. A., Int. J. Heat Mass Transfer 26:1265 (1983).

14. Sangiovanni, J. J., and Kesten, A. S., Combust. Sci. Tech. 16:59 (1977).

15. Sangiovanni, J. J., and Labowsky M., Combust. Flame 47:15 (1982).

16. Schaub, S. A., Alexander D. R., and Barton J. P., Appl. Opt. 33:473-483 (1994).

17. Berglund, R. N., and Liu, B. Y. H., Environ. Sci. Technol. 7(2):147-153 (1973).

18. Cho, S. Y., Choi, M. Y., and Dryer F. L., Twenty-Third Symposium (International) on Combustion, The Combustion Institute, Pittsburgh, 1990, p. 1611.

19. Jiang, T. L., Chen, Wei S., Ming Jiung T., and Chiu Huei H., Combust. Flame 103:221 (1995).

20. Bowman, C. T., Combust. Flame 25:343 (1975).

21. Curran, H. J., Dunphy, M. P., Simmie, J. M., Westbrook, C. K., and Pitz, W. J., Twenty-Fourth Symposium (International) on Combustion, The Combustion Institute, Pittsburgh, 1992, p. 769.

22. Li, S. C., Libby, P. A., and Williams, F. A., TwentyFourth Symposium (International) on Combustion, The Combustion Institute, Pittsburgh, 1992, p. 1503.

23. Marchese, A. J., and Dryer, F. D., Combust. Flame 105:104 (1996).

24. Zhang, B. L., and Williams, F. A., Acta Astronautica 39:599(1997).

Received 16 November 2001; revised 4 March 2002; accepted 25 March 2002 